Pacific

Journal of

Mathematics

\title{
INTEGER POINTS ON ELLIPTIC CURVES
}

Wen-Chen Chi, King Fai Lai and Ki-Seng Tan 


\title{
INTEGER POINTS ON ELLIPTIC CURVES
}

\author{
Wen-Chen Chi, King Fai Lai And Ki-Seng Tan
}

\begin{abstract}
We study Lang's conjecture on the number of $S$-integer points on an elliptic curve over a number field. We improve the exponent of the bound of Gross and Silverman from quadratic to linear by using the $S$-unit equation method of Evertse and a formula on 2-division points.
\end{abstract}

\section{Introduction}

Let $E$ be an elliptic curve defined over an algebraic number field $k$ of degree $d$. For a finite set $S$ of places of $k$ containing all the archimedean ones, we denote the ring of $S$-integers of $k$ by $0_{S}$. Serge Lang conjectured that if the Weierstrass equation of $E$ is quasiminimal, then the cardinality of the set $E\left(O_{S}\right)$ of $O_{S}$-integer points of $E$ should be bounded in terms of the field $k$, the cardinality of $S$ and the rank of the group $E(k)$ of $k$-rational points of $E$ [Lang 1978, p. 140]. Silverman [1987] proved Lang's conjecture when $E$ has integral $j$-invariant. In general, if $j(E)$ is nonintegral for at most $\delta$ places of $k$, then a bound was also given with $\delta$ involved. However he did not compute the constants involved. Gross and Silverman [1995] used Roth's theorem to obtain an explicit bound. To state their theorem, let us write the Weierstrass equation of the elliptic curve $E$ as

$$
Y^{2}=X^{3}+\mathscr{A} X+\mathscr{B},
$$

where $\mathscr{A}, \mathscr{B} \in \mathscr{O}_{S}$. Put $\Delta=4 \mathscr{A}^{3}+27 \mathscr{B}^{2}$. Write $j(E)$ for the $j$-invariant of $E$. Let $D_{k}$ and $R_{k}$ be the discriminant and the regulator of $k$. Let $M_{k}$ be the set of all places of $k$. For a place $v \in M_{k}$, let $k_{v}$ be the completion of $k$ at $v$ and let $\mid l_{v}$ be such that, for $z \in \mathbb{Q}$,

$$
|z|_{v}=|z|_{p}^{\left[k_{v}: \mathbb{Q}_{p}\right] /[k: \mathbb{Q}]}
$$

MSC2000: primary 11D45; secondary 11G05, 14K12.

Keywords: elliptic curves, $S$-integers, integer points, $S$-unit equations, 2-division points, Lang's conjecture.

Chi and Tan were supported in part by the National Science Council of Taiwan, grants NSC91-2115M-003-006 and NSC89-2115-M-002-003 respectively. 
where $p$ is the place of $\mathbb{Q}$ lying under $v$ and ||$_{p}$ is the usual absolute value. We use $h_{k}$ to denote the multiplicative height. Namely, for $x \in k$

$$
h_{k}(x)=\prod_{v \in M_{k}} \max \left(|x|_{v}, 1\right) .
$$

We shall write $s$ for the cardinality of the set $S$.

Theorem 1.1 [1995]. Suppose that (1-1) is quasiminimal and that

$$
6 d\left(60 d^{2} \log 6 d\right)^{d}\left(\frac{2}{\sqrt{3}}\right)^{d(d-1) / 2} \cdot \max \left(R_{k}, \log \left|D_{k}\right|, 1\right) .
$$

is at most

$$
\max \left\{\log h_{k}(j(E)), \log \left|\operatorname{Norm}_{k / \mathbb{Q}}(\Delta)\right|\right\} .
$$

Then

$$
\# E\left(\mathscr{O}_{S}\right) \leq 2 \cdot 10^{11} \cdot d \cdot \delta^{3 d} \cdot\left(32 \cdot 10^{9}\right)^{r \delta+s} .
$$

In this paper, we take a completely different approach. By using a formula on 2-division points from [2002], we associate to an $S$-integer point an unit equation over an extension of $k$. Then we use the machinery developed by J.-H. Evertse [1984] to obtain a quantitative bound for the number of $S$-integer points. Let $\mathscr{D}_{E / k}$ be the ideal of the minimal discriminant of $E / k$. Then we have

$$
(\Delta)=\mathscr{D}_{E / k} \cdot \prod_{v} P_{v}^{12 \chi_{v}}
$$

where $P_{v}$ is the prime ideal corresponding to the place $v$ and $\chi_{v} \in \mathbb{Z}$. For $v \in S$, $\chi_{v} \geq 0$. We factor the cubic over the algebraic closure $\bar{k}$ of $k$ as

$$
X^{3}+\mathscr{A} X+\mathscr{B}=(X-\alpha)(X-\beta)(X-\gamma) .
$$

Let $k_{1}=k(\alpha, \beta, \gamma)$ and $m=\left[k_{1}: k\right]$. Further, let $M_{k, 0}$ be the set of all nonarchimedean places in $k$.

Definition 1.2. Let $w$ be a nonarchimedean place over a field extension $K / k_{1}$. If the valuations $w(\alpha-\beta), w(\beta-\gamma), w(\gamma-\alpha)$ are all equal, we say that $E$ has $G$-type reduction at $w$; otherwise, we say that $E$ has $M$-type reduction at $w$.

In fact, if $w^{\prime}$ is another place of $K$ such that both $w$ and $w^{\prime}$ are sitting over a place $v \in M_{k, 0}$, then the reductions of $E$ at $w$ and $w^{\prime}$ are of the same type. Therefore, we will say that at $v$, the reduction of $E$ is also of that type. Furthermore, in the case where $v(2)=0, E$ has G-type reduction if and only if it has good or potential good reduction (see Lemma 3.1). 
Define

$$
\begin{aligned}
S_{0} & =\left\{v \in M_{k, 0} \backslash S \mid v(2)=0, \chi_{v}=0, v(\Delta)>0, v(j(E)) \geq 0\right\}, \\
S_{1} & =\left\{v \in M_{k, 0} \mid \chi_{v}>0, v(j(E)) \geq 0\right\}, \\
S_{m} & =\left\{v \in M_{k, 0} \mid E \text { has M-type reduction at } v\right\}, \\
S^{\prime} & =S \backslash\left(S_{0} \cup S_{1} \cup S_{m}\right) .
\end{aligned}
$$

Let $s_{1}, s_{m}, s^{\prime}$ be the cardinality of $S_{1}, S_{m}, S^{\prime}$. Then $s_{m}$ is at most $\delta+d$.

With the notations above, we can now state our main result.

\section{Theorem 1.3.}

$$
\# E\left(\mathbb{O}_{S}\right) \leq 11 \times 7^{1.64 r+2.27\left(s^{\prime}+s_{1}\right)+3.7 s_{m}+10.3 m d} .
$$

Note that we do not require the equation (1-1) to be quasiminimal. If we did so, then, by [Silverman 1984, p. 238], we would have

$$
\left|\operatorname{Norm}_{k / \mathbb{Q}} \prod_{v \in S_{1}} P^{\chi_{v}}\right| \leq\left|D_{k}\right|^{6},
$$

and hence

$$
s_{1} \leq 6 \log \left|D_{k}\right| .
$$

The exponent in the Gross-Silverman bound is quadratic in $\delta$ and $r$, while ours is linear, and our constants are smaller. Also, if the ABC Conjecture holds, our method can be applied to get a bound only in terms of $r$ and $k$, in which the exponent is linear in $s$ and $r$ and differs from that obtained in [Hindry and Silverman 1988]. In fact, this has been achieved in [Chi et al. 2004] for the case where $k$ is a function field of characteristic zero. Also, the method can be modified to bound the number of integer solutions to $Y^{n}=F(X)$; see [Chi et al. $\geq 2006$ ].

\section{A formula for 2-division points}

The following result can be proved by straightforward calculations. For details, see [Tan 2002] or [Chi et al. 2004, Section 2.2].

Lemma 2.1. In the notations preceding Theorem 1.3 a point $P=(a, b) \in E(k)$ determines an extension

$$
K=k_{1}(\sqrt{a-\alpha}, \sqrt{a-\beta}, \sqrt{a-\gamma})
$$

depending only on the class $[P] \in E(k) / 2 E(k)$. Given a choice of signs for $\sqrt{a-\alpha}$, $\sqrt{a-\beta}$, and $\sqrt{a-\gamma}$ such that

$$
b=\sqrt{a-\alpha} \sqrt{a-\beta} \sqrt{a-\gamma},
$$


the point $Q:=(f, g) \in E(K)$ defined by

$$
f-\alpha=(\sqrt{a-\alpha}+\sqrt{a-\beta})(\sqrt{a-\alpha}+\sqrt{a-\gamma}),
$$

and

$$
g=(\sqrt{a-\alpha}+\sqrt{a-\beta})(\sqrt{a-\beta}+\sqrt{a-\gamma})(\sqrt{a-\gamma}+\sqrt{a-\alpha}),
$$

satisfies

$$
2 Q=P \text {. }
$$

Furthermore, if $\left\{\alpha_{1}, \alpha_{2}, \alpha_{3}\right\}=\{\alpha, \beta, \gamma\}, D_{i}=\left(\alpha_{i}, 0\right) \in E\left(k_{1}\right), i=1,2,3$, and $Q^{(i)}=\left(f^{(i)}, g^{(i)}\right)=Q+D_{i}$, then

$$
\left(f-\alpha_{i}\right)\left(f^{(i)}-\alpha_{i}\right)=\left(\alpha_{i}-\alpha_{j}\right)\left(\alpha_{i}-\alpha_{j^{\prime}}\right),
$$

where $\left\{j, j^{\prime}\right\}=\{1,2,3\} \backslash\{i\}$.

\section{Local calculations}

Given a point $P \in E(k)$, let $K$ be the field determined by $P$ as in Lemma 2.1. For $v \in M_{k}$, let $K_{w}$ be the completion of $K$ with respect to a place $w$ lying over $v$. Then $K_{w} / k_{v}$ is a Galois extension. Let $I_{w}$ be the inertia subgroup of $\operatorname{Gal}\left(K_{w} / k_{v}\right)$. In this section, we assume that $w$ is nonarchimedean and view it as an valuation from $K_{w}$ onto $\mathbb{Z} \cup\{\infty\}$.

Lemma 3.1. Suppose $E$ has potential good reduction at a place $v$ of $k$ such that $v(2)=0$. Then for any place $w$ of $K$ lying over $v$, we have

$$
w(\alpha-\beta)=w(\beta-\gamma)=w(\gamma-\alpha) .
$$

Proof. Suppose on the contrary that

$$
w(\gamma-\alpha)>w(\alpha-\beta)=w(\beta-\gamma)
$$

We can find a field extension $\tilde{K}$ of $K$ such that $\tilde{v}(\alpha-\beta)=2 m, m \in \mathbb{Z}$, where $\tilde{v}$ is a place of $\tilde{K}$ lying over $w$. By our assumption, we have $\tilde{v}(\beta-\gamma)=2 m$ and $\tilde{v}(\gamma-\alpha)>2 m$. Consider the elliptic curve $\tilde{E}$ defined by

$$
\tilde{E}: \quad \tilde{Y}^{2}=\tilde{X}(\tilde{X}-\tilde{\beta})(\tilde{X}-\tilde{\gamma}),
$$

which was obtained from (1-1) by the change of variables

$$
\begin{array}{ll}
\tilde{Y}=Y / \pi^{3 m}, & \tilde{X}=(X-\alpha) / \pi^{2 m}, \\
\tilde{\beta}=(\beta-\alpha) / \pi^{2 m}, & \tilde{\gamma}=(\gamma-\alpha) / \pi^{2 m},
\end{array}
$$


where $\pi$ is a uniformizer of the prime ideal associated to $\tilde{v}$ in $\tilde{K}$. Then $\tilde{v}(\tilde{\beta})=0$ and $\tilde{v}(\tilde{\gamma})>0$. This implies that $\tilde{E}$ has multiplicative reduction at $\tilde{v}$. Consequently, $\tilde{v}\left(j_{E}\right)=\tilde{v}\left(j_{\tilde{E}}\right)<0$ which contradicts our hypothesis.

Now assume that the equation for $E$ is minimal at $v$. Let $\mathbb{F}_{v}$ be the residue field of $v$ and let $\bar{E}$ be the reduction of $E$ at $v$. As usual, for $P \in E\left(k_{v}\right)$, we denote its image under the reduction map $E\left(k_{v}\right) \rightarrow \bar{E}\left(\mathbb{F}_{v}\right)$ by $\bar{P}$. Put

$$
E_{0}\left(k_{v}\right)=\left\{P \in E\left(k_{v}\right) \mid \bar{P} \in \bar{E}_{n s}\left(\mathbb{F}_{v}\right)\right\},
$$

where $\bar{E}_{n s}$ is the set of nonsingular points of $\bar{E}$. We have the following key lemma. Here we retain the notations in Lemma 2.1.

Lemma 3.2. Assume that at $v$, where $v(2)=0$, the Weierstrass equation (1-1) is minimal and $E$ has potential good reduction. For $P_{1}, P_{2} \in E\left(O_{v}\right)$, let $Q_{i}=$ $\left(f_{i}, g_{i}\right) \in E\left(K_{w}\right)$, for $i=1,2$, be such that $2 Q_{i}=P_{i}$. If $Q_{1}-Q_{2} \in E_{0}\left(k_{v}\right)$, then

$$
w\left(f_{1}-\alpha\right)=w\left(f_{2}-\alpha\right) \text { and } \quad w\left(f_{1}-\beta\right)=w\left(f_{2}-\beta\right) .
$$

Before we give the proof of Lemma 3.2, we recall some basic facts on the formal group associated to an elliptic curve.

Suppose $w(\alpha-\beta)=2 a+\epsilon$, where $a \in \mathbb{N} \cup\{0\}$ and $\epsilon=0$ or 1. By Lemma 3.1, $w(\beta-\gamma)=w(\gamma-\alpha)=2 a+\epsilon$. Consider the change of variables

$$
\begin{array}{ll}
\tilde{Y}=Y / \pi^{3 a}, & \tilde{X}=(X-\alpha) / \pi^{2 a}, \\
\tilde{\beta}=(\beta-\alpha) / \pi^{2 a}, & \tilde{\gamma}=(\gamma-\alpha) / \pi^{2 a},
\end{array}
$$

where $\pi$ is a uniformizer of the prime ideal associated to $w$. Then

$$
\tilde{E}: \tilde{Y}^{2}=\tilde{X}(\tilde{X}-\tilde{\beta})(\tilde{X}-\tilde{\gamma}),
$$

is a minimal Weierstrass equation for $E$ over $K_{w}$. For $i=1,2$, let $\tilde{Q}_{i}=\left(\tilde{f}_{i}, \tilde{g}_{i}\right)$, be the points on $\tilde{E}$ corresponding to $Q_{i}$. Let $\hat{E}$ be the formal group associated to $\tilde{E} / K_{w}$. For $m \geq 0$, set

$$
\hat{E}_{m}= \begin{cases}\tilde{E}_{0}\left(K_{w}\right) & \text { if } m=0, \\ \hat{E}\left(\pi^{m} \mathcal{O}_{K_{w}}\right) & \text { if } m>0 .\end{cases}
$$

Then we have the filtration

$$
\cdots \subset \hat{E}_{m+1} \subset \hat{E}_{m} \subset \cdots \subset \hat{E}_{1} \subset \hat{E}_{0} .
$$

Also, recall that we have the exact sequence

$$
0 \longrightarrow \hat{E}_{1} \longrightarrow \hat{E}_{0} \longrightarrow \overline{\tilde{E}}_{n s} \longrightarrow 0
$$

where $\overline{\tilde{E}}_{n s}$ is the nonsingular part of the reduction of $\tilde{E}$. 
For a point $R=(\tilde{X}, \tilde{Y})$ in $\tilde{E}\left(K_{w}\right)$, let $\tilde{t}=-\tilde{X} / \tilde{Y}$. The following lemma follows easily from [Silverman 1986, Chapter IV].

Lemma 3.3. Let notations be as above.

(1) If $m>0$, then

$$
R \in \hat{E}_{m} \backslash \hat{E}_{m+1} \Longleftrightarrow w(\tilde{t})=m \Longleftrightarrow(w(\tilde{X})=-2 m \text { and } w(\tilde{Y})=-3 m) .
$$

(2) If $m=0$ and $\epsilon=0$, then

$$
R \in \hat{E}_{0} \backslash \hat{E}_{1} \Longleftrightarrow w(\tilde{t}) \leq 0 \Longleftrightarrow(w(\tilde{X}) \geq 0 \text { and } w(\tilde{Y}) \geq 0) .
$$

(3) If $m=0$ and $\epsilon=1$, then

$$
R \in \hat{E}_{0} \backslash \hat{E}_{1} \Longleftrightarrow w(\tilde{t})=0 \Longleftrightarrow(w(\tilde{X})=0 \text { and } w(\tilde{Y})=0) .
$$

Note that if $\epsilon=0$, then $\tilde{E}$ has good reduction at $w$. In this case, $\hat{E}_{0}=\tilde{E}\left(K_{w}\right)$.

Lemma 3.4. Under the hypothesis of Lemma 3.2, suppose that $w(\alpha-\beta)=2 a+\epsilon$ and $Q=(f, g) \in E_{0}\left(k_{v}\right)$. Then $\tilde{Q} \in \hat{E}_{a} \subset \hat{E}_{0}$.

Proof. Recall that the reduction of $E$ is

$$
\bar{E}: \bar{Y}^{2}=(\bar{X}-\bar{\alpha})(\bar{X}-\bar{\beta})(\bar{X}-\bar{\gamma}) .
$$

The singularity of $\bar{E}$ is $(\bar{\alpha}, 0)$.

If $Q=(f, g) \in E_{0}\left(k_{v}\right)$, then $w(f-\alpha) \leq 0$. Since $\tilde{f}=(f-\alpha) / \pi^{2 a}, \tilde{g}=g / \pi^{3 a}$, we have $w(\tilde{f}) \leq-2 a$. By Lemma 3.3, we have $\tilde{Q} \in \hat{E}_{a} \subset \hat{E}_{0}$.

Proof of Lemma 3.2. We apply Lemma 2.1 with $\alpha_{1}=\alpha, \alpha_{2}=\beta$, and $\alpha_{3}=\gamma$. Then $Q_{1}^{\prime}=Q_{1}+(\alpha, 0)$, and so on. By (2-1), we have

$$
\left(f_{1}-\alpha\right)\left(f_{1}^{\prime}-\alpha\right)=(\alpha-\beta)(\alpha-\gamma) .
$$

This and Lemma 3.1 imply

$$
w\left(f_{1}-\alpha\right)+w\left(f_{1}^{\prime}-\alpha\right)=2(2 a+\epsilon),
$$

and

$$
w\left(\tilde{f}_{1}\right)+w\left(\tilde{f}_{1}^{\prime}\right)=2 \epsilon
$$

Similarly,

$$
w\left(\tilde{f}_{2}\right)+w\left(\tilde{f}_{2}^{\prime}\right)=2 \epsilon .
$$

First we consider the case where

$$
w\left(f_{1}-\alpha\right) \leq 2 a+\epsilon .
$$


Then $w\left(\tilde{f}_{1}\right) \leq \epsilon$. If $w\left(\tilde{f}_{1}\right)>0$, then $w\left(\tilde{f}_{1}\right)=\epsilon=1$. In this situation, $\tilde{E}$ has additive reduction at $w$ and $(0,0)$ is the singularity of the reduction. Therefore, $\tilde{Q}_{1} \notin \tilde{E}_{0}\left(K_{w}\right)$. By Lemma 3.4, $\tilde{Q}_{1}-\tilde{Q}_{2} \in \hat{E}_{a} \subset \hat{E}_{0}$, and consequently $\tilde{Q}_{2}$ is not in $\tilde{E}_{0}\left(K_{w}\right)$. Hence $w\left(\tilde{f}_{2}\right)>0$. By $(3-1)$, we also have $w\left(\tilde{f}_{1}^{\prime}\right)=1$. Repeating the above argument, we also conclude that $w\left(\tilde{f}_{2}^{\prime}\right)>0$. Then (3-2) implies that $w\left(\tilde{f}_{2}\right)=w\left(\tilde{f}_{2}^{\prime}\right)=1$.

Now, assume that $w\left(\tilde{f}_{1}\right)=-2 m \leq 0$. Note that by Lemma $2.1 Q_{i} \in E\left(\mathscr{O}_{w}\right)$, $i=1,2$ and we have $w\left(f_{i}-\alpha\right) \geq 0$. Hence,

$$
w\left(\tilde{f}_{i}\right) \geq-2 a .
$$

This means that $\tilde{Q}_{1} \notin \hat{E}_{a+1}$ and $\tilde{Q}_{1} \in \hat{E}_{m} \backslash \hat{E}_{m+1}$. If $a>m$, then by Lemma 3.3 and Lemma 3.4, we also have

$$
\tilde{Q}_{2} \in \hat{E}_{m} \backslash \hat{E}_{m+1}
$$

and hence $w\left(\tilde{f}_{2}\right)=-2 m$. If $a=m$, then we have $\tilde{Q}_{2} \in \hat{E}_{a}$ and hence $w\left(\tilde{f}_{2}\right) \leq-2 a$. By (3-3), we have $w\left(\tilde{f}_{2}\right)=-2 m$, too.

For the case where

$$
w\left(f_{1}-\alpha\right)>2 a+\epsilon,
$$

we consider $f_{1}^{\prime}$, which, according to (2-1), satisfies

$$
w\left(f_{1}^{\prime}-\alpha\right)<2 a+\epsilon .
$$

Then the argument above can be applied to verify that

$$
w\left(f_{2}^{\prime}-\alpha\right)=w\left(f_{1}^{\prime}-\alpha\right) .
$$

We complete the proof by applying (2-1).

Let $K$ be as given in Lemma 2.1 and let $w$ be a nonarchimedean place of $K$. A point $Q=(f, g) \in E\left(K_{w}\right)$ is called special if

$$
w(f-\alpha)<\min \{w(\alpha-\beta), w(\beta-\gamma), w(\gamma-\alpha)\} .
$$

If $Q$ is special, then

$$
w(f-\alpha)=w(f-\beta)=w(f-\gamma) .
$$

Put $\left\{\alpha_{1}, \alpha_{2}, \alpha_{3}\right\}=\{\alpha, \beta, \gamma\}$, and let $Q^{(i)}$ be as in Lemma 2.1.

Lemma 3.5. Suppose that $Q^{(0)}=Q \in E\left(K_{w}\right)$ and $E$ has $G$-type reduction at $w$ with

$$
w\left(\alpha_{1}-\alpha_{2}\right)=w\left(\alpha_{2}-\alpha_{3}\right)=w\left(\alpha_{3}-\alpha_{1}\right)=\epsilon
$$


(1) If $Q$ is special and $w\left(f-\alpha_{1}\right)=\epsilon-e<\epsilon$, then for $j=1,2,3, Q^{(j)}$ is not special and

$$
w\left(f^{(j)}-\alpha_{i}\right)= \begin{cases}\epsilon+e & \text { if } i=j, \\ \epsilon & \text { if } i \neq j .\end{cases}
$$

(2) If every $Q^{(j)}$ is not special for $j=0,1,2,3$, then, for every $i$ and $j$,

$$
w\left(f^{(j)}-\alpha_{i}\right)=\epsilon .
$$

Proof. Suppose that $Q$ is special. By (2-1),

$$
w\left(f^{(j)}-\alpha_{j}\right)=2 w(\alpha-\beta)-w(f-\alpha)=\epsilon+e .
$$

If $i \neq j$, then

$$
w\left(f^{(j)}-\alpha_{i}\right)=w\left(f^{(j)}-\alpha_{j}+\alpha_{j}-\alpha_{i}\right)=\min (\epsilon+e, \epsilon)=\epsilon .
$$

If every $Q^{(j)}, j=0,1,2,3$, is not special, then for every $i, w\left(f^{(j)}-\alpha_{i}\right) \geq \epsilon$. By $(2-1)$ again, we must have $w\left(f^{(j)}-\alpha_{i}\right) \leq \epsilon$.

Lemma 3.6. Suppose that $Q \in E\left(K_{w}\right)$ and $E$ has M-type reduction with

$$
\epsilon_{1}=w\left(\alpha_{1}-\alpha_{2}\right)=w\left(\alpha_{1}-\alpha_{3}\right)<w\left(\alpha_{2}-\alpha_{3}\right)=\epsilon_{2} .
$$

(1) If $Q$ is special and $w\left(f-\alpha_{1}\right)=\epsilon_{1}-e<\epsilon_{1}$, then, for $j=1,2,3, Q^{(j)}$ is not special and

$$
w\left(f^{(j)}-\alpha_{i}\right)= \begin{cases}\epsilon_{1}+e & \text { if } i=j=1, \\ \epsilon_{2}+e & \text { if } i=j=2,3 \\ \epsilon_{1} & \text { if }(j=1, i \neq 1) \text { or }(i=1, j \neq 1), \\ \epsilon_{2} & \text { if } i, j=2,3, j \neq i .\end{cases}
$$

(2) If every $Q^{(j)}, j=0,1,2,3$, is not special and $w\left(f-\alpha_{2}\right)=\epsilon_{1}+e$, then

$$
\epsilon_{1}=w\left(f-\alpha_{1}\right) \leq \epsilon+e=w\left(f-\alpha_{3}\right) \leq \epsilon_{2} .
$$

Moreover, for $i, j=1,2,3$,

$$
w\left(f^{(j)}-\alpha_{i}\right)= \begin{cases}\epsilon_{1}+e & \text { if } j=1, i \neq 1 \\ \epsilon_{1} & \text { if } i=1 \\ \epsilon_{2}-e & \text { if } i \neq 1, j \neq 1 .\end{cases}
$$

Proof. Most of the proof is similar to that of Lemma 3.5. Only the valuations of $f^{(1)}-\alpha_{i}, i \neq 1$, need special calculation. But, since $Q^{(1)}=Q^{(2)}+D_{3}$ and 
$Q^{(1)}=Q^{(3)}+D_{2}$, by (2-1), we have

$$
\begin{aligned}
& w\left(f^{(2)}-\alpha_{2}\right)+w\left(f^{(1)}-\alpha_{2}\right)=\epsilon_{1}+\epsilon_{2}, \\
& w\left(f^{(3)}-\alpha_{3}\right)+w\left(f^{(1)}-\alpha_{3}\right)=\epsilon_{1}+\epsilon_{2} .
\end{aligned}
$$

\section{Unit equations}

Let

$$
\mathscr{C}=\left\{(P, Q) \mid P \in E\left(\mathcal{O}_{S}\right), 2 Q=P\right\} .
$$

For $\left(P_{1}, Q_{1}\right),\left(P_{2}, Q_{2}\right) \in \mathscr{C}$, we define an equivalence relation as follows:

$$
\left(P_{1}, Q_{1}\right) \sim\left(P_{2}, Q_{2}\right) \text { if and only if } Q_{1}-Q_{2} \in 12 E(k) .
$$

Let $\left(P_{1}, Q_{1}\right), \ldots,\left(P_{c}, Q_{c}\right)$ represent all the equivalence classes in $\mathscr{C}_{\text {. Then }}$

$$
c \leq 4 \times E(k) / 24 E(k) \leq 4 \times 24^{r+2} .
$$

Now, we fix an equivalence class represented by $\left(P_{l}, Q_{l}\right)$. If $(P, Q) \sim\left(P_{l}, Q_{l}\right)$ and $Q=(f, g), Q_{l}=\left(f_{l}, g_{l}\right)$, then the quantities

$$
\begin{array}{ll}
x=(f-\alpha) /\left(f_{l}-\alpha\right), & y=(f-\beta) /\left(f_{l}-\beta\right), \\
\lambda=\left(f_{l}-\alpha\right) /(\beta-\alpha), & \mu=\left(\beta-f_{l}\right) /(\beta-\alpha)
\end{array}
$$

satisfy

$$
\lambda x+\mu y=1 .
$$

Note that $Q$ and $Q_{l}$ determine the same field extension $K / k$. Let

$$
\tilde{S}=\left\{w \mid w \in M_{K} \text { and } w \mid v, \text { for some } v \in S^{\prime} \cup S_{1} \cup S_{m}\right\} .
$$

Using (2-1), we see that $x$ and $y$ are units at every place $w$ not sitting over $S \cup$ $S_{0} \cup S_{1} \cup S_{m}$. For $v \in S_{0}, E$ has additive reduction at $v$. Therefore,

$$
12 E\left(k_{v}\right) \subset E_{0}\left(k_{v}\right) .
$$

Applying Lemma 3.2 to $Q$ and $Q_{l}$, we see that (4-2) is an $\tilde{S}$-unit equation.

Now we apply the theory of [Evertse 1984] to bound the cardinality of the equivalence class of $\left(P_{l}, Q_{l}\right)$. We will follow the setting in that paper. Fix a primitive third root $\rho$ of 1 and put $L=K(\rho)$. Given $(P, Q)$ in the equivalence class of $\left(P_{l}, Q_{l}\right)$, we define $x, y, \lambda, \mu$ by $(4-1)$ and put

$$
\xi=\xi(x, y)=\lambda x-\rho \mu y, \eta=\eta(x, y)=\lambda x-\rho^{2} \mu y, \zeta=\zeta(x, y)=\xi / \eta .
$$

We denote by $\mathscr{q}^{0}$ the set of those $\zeta \in L$ for which an $\tilde{S}$-unit solution $(x, y)$ of (4-2) exists with $\lambda x / \mu y$ not a root of one and such that $\zeta=\zeta(x, y)$. We denote by $\mathscr{V}^{1}$ the subset consisting of those $\zeta(x, y)$ such that $x$ and $y$ are defined by (4-1) 
using a point $(P, Q)$ in the equivalence class of $\left(P_{l}, Q_{l}\right)$. We can recover $x$ and $y$ from $\zeta$. Therefore, it is enough to bound the number of elements in $\mathscr{V}^{1}$.

Let $T$ be the set of places of $L$ sitting over $\tilde{S}$ and put

$$
A=\left(\prod_{V \in T}|3|_{V}\right)^{1 / 2} \prod_{V \in T}|\lambda \mu|_{V}\left(\prod_{V \notin T} \max \left(|\lambda|_{V} \cdot|\mu|_{V}\right)\right)^{3} .
$$

Definition 4.1. For $V \in M_{L}, \zeta \in L$, put

$$
m_{V}(\zeta)=\min _{i=0,1,2}\left(1, \max \left(\left|1-\rho^{i} \zeta\right|_{V},\left|1-\rho^{-i} \zeta^{-1}\right|_{V}\right)\right.
$$

Lemma 4.2 [Evertse 1984, Lemma 3]. We have

$$
\prod_{V \in T} m_{V}(\zeta) \leq 8 A h(\zeta)^{-3} \quad \text { for } \zeta \in \mathscr{V}^{0}
$$

The next lemma follows by direct calculation.

Lemma 4.3. Suppose that $V \in M_{L}$ is nonarchimedean and $\zeta=\zeta(x, y) \in \mathscr{V}^{0}$.

(1) If $|\mu y|_{V}<1$, then

$$
\begin{aligned}
m_{V}(\zeta) & =|1-\zeta|_{V}=|(1-\rho) \mu y|_{V} \\
& <\left|1-\rho^{i} \zeta\right|_{V}, \text { for } i \neq 0 .
\end{aligned}
$$

(2) If $|\lambda x|_{V}<1$, then

$$
\begin{aligned}
m_{V}(\zeta) & =|1-\rho \zeta|_{V}=|(1-\rho) \lambda x|_{V} \\
& <\left|1-\rho^{i} \zeta\right|_{V}, \text { for } i \neq 1 .
\end{aligned}
$$

(3) If $|\lambda x|_{V}^{-1}<1$, then

$$
\begin{aligned}
m_{V}(\zeta) & =\left|1-\rho^{2} \zeta\right|_{V}=\left|(1-\rho)(\lambda x)^{-1}\right|_{V} \\
& <\left|1-\rho^{i} \zeta\right|_{V}, \text { for } i \neq 2 .
\end{aligned}
$$

(4) If $|\lambda x|_{V}=|\mu y|_{V}=1$, then

$$
\begin{aligned}
m_{V}(\zeta) & =|1-\zeta|_{V}=|1-\rho \zeta|_{V} \\
& =\left|1-\rho^{2} \zeta\right|_{V}=|1-\rho|_{V} .
\end{aligned}
$$

Definition 4.4. For a $\zeta$ in $\mathscr{V}^{0}$ and $V \in T$, we choose a $\rho_{V} \in\left\{1, \rho, \rho^{2}\right\}$ such that

$$
m_{V}(\zeta)=\min \left(1, \max \left(\left|1-\rho_{V} \zeta\right|_{V},\left|1-\rho_{V}^{-1} \zeta^{-1}\right|_{V}\right)\right) .
$$

If $V$ is nonarchimedean and we are in case (4) of the preceding lemma, we choose $\rho_{V}=1$. 
For a nonarchimedean place $v \in S^{\prime} \cup S_{1} \cup S_{m}$, let

$$
T_{v}=\{V \in T|V| v\} .
$$

Recall that if $\zeta \in \mathscr{V}^{1}$, there is an associated $(P, Q) \in \mathscr{C}$.

From now on, we fix the indices so that $\alpha_{1}=\alpha, \alpha_{2}=\beta, \alpha_{3}=\gamma, D_{i}=\left(\alpha_{i}, 0\right)$, and as before, we put $Q^{(i)}=Q+D_{i}$.

Definition 4.5. Let $\zeta$ be in $\mathscr{V}^{1}$ and let $V$ be a nonarchimedean place. We say that $\zeta$ is of type $i$, where $i=0,1,2,3$, if $Q^{(i)}$ is special at $V$. If none of the $Q^{(i)}$ is special, we say that $\zeta$ is of type 4 .

Consider the set of numbers

$$
\left|\left(f^{(j)}-\alpha_{j_{1}}\right) /\left(\alpha_{j_{1}}-\alpha_{j_{2}}\right)\right|_{V}
$$

and their inverses, where we take $j=0,1,2,3, j_{1}, j_{2}=1,2,3$, and $j_{1} \neq j_{2}$. By the conductor of $\zeta$ at $V$ we mean the set $C_{V}(\zeta)$ consisting of all those numbers in this set which are at most one. We list the elements of $C_{V}(\zeta)$ as $c_{V, i}$ with $i=0,1,2, \ldots$ and $c_{V, 0}=1$. If $E$ has G-type reduction at $V$, then Lemma 3.5 implies that

$$
C_{V}= \begin{cases}\left\{1, c_{V, 1}\right\} & \text { if } \zeta \text { is of type } 0,1,2,3 ; \\ \{1\} & \text { if } \zeta \text { is of type } 4 .\end{cases}
$$

Also, if $E$ has M-type reduction at $V$, then Lemma 3.6 implies that

$$
C_{V}= \begin{cases}\left\{1, c_{V, 1}, c_{V, 2}\right\} & \text { if } \zeta \text { is of type } 0,1,2,3 ; \\ \left\{1, c_{V}\right\} \text { or }\left\{1, c_{V, 1}, c_{V, 2}\right\} & \text { if } \zeta \text { is of type } 4 .\end{cases}
$$

Set $\mathscr{G}=\operatorname{Gal}(L / k)$. Then $\mathscr{G}$ acts transitively on $T_{v}$ and for $z \in L, \sigma \in \mathscr{G}$, we have

$$
|z|_{\sigma(V)}=\left|\sigma^{-1}(z)\right|_{V}
$$

For $z=(f-\alpha) /(\alpha-\beta)$, or $z=(f-\beta) /(\alpha-\beta)$, we have

$$
\sigma^{-1}(z) \in\left\{\left(f^{(j)}-\alpha_{i}\right) /\left(\alpha_{i}-\alpha_{i^{\prime}}\right) \mid j=0,1,2,3, i, i^{\prime}=1,2,3\right\} .
$$

From these facts and Lemma 4.3, we can deduce the next result:

Lemma 4.6. Let $v \in S^{\prime} \cup S_{1} \cup S_{m}$ be a nonarchimedean place and let $V_{0}$ be a place in $T_{v}$. Then, for a given $\zeta \in \mathcal{V}^{1}$, the map $T_{v} \rightarrow\left\{1, \rho, \rho^{2}\right\}, V \mapsto \rho_{V}$, depends only on the type of $\zeta$ at $V_{0}$. Moreover, if $E$ has $G$-type reduction at $v$ and $C_{V_{0}}=\{1\}$ or $\left\{1, c_{V_{0}, 1}\right\}$, there is a decomposition

$$
T_{v}=T_{v}^{0} \cup T_{v}^{1},
$$


which depends only on the type of $\zeta$ such that

$$
m_{V}= \begin{cases}1 & \text { if } V \in T_{v}^{0} \\ c_{V_{0}, 1} & \text { if } V \in T_{v}^{1} .\end{cases}
$$

Also, if $E$ has M-type reduction at $v$, there is a decomposition

$$
T_{v}=T_{v}^{0} \cup T_{v}^{1} \cup T_{v}^{2},
$$

which depends only on the type of $\zeta$ such that

$$
m_{V}= \begin{cases}1 & \text { if } V \in T_{v}^{0}, \\ c_{V_{0}, 1} & \text { if } V \in T_{v}^{1}, \\ c_{V_{0}, 2} & \text { if } V \in T_{v}^{2} .\end{cases}
$$

Let $v \in S^{\prime} \cup S_{1} \cup S_{m}$ be a nonarchimedean place. We fix a place $V_{0}$ in $T_{v}$, and put $t_{v}^{i}=\# T_{v}^{i}$. If $E$ has G-type reduction at $v$, define

$$
m_{v}=c_{V_{0}, 1}^{t_{v}^{1}} .
$$

If $E$ has M-type reduction at $v$, define

$$
m_{v, 1}=c_{V_{0}, 1}^{t_{v}^{1}} \quad \text { and } \quad m_{v, 2}=c_{V_{0}, 2}^{t_{v}^{2}}
$$

Here we use the convention that if $T_{v}^{i}$ is empty, the associated $m_{v}$ or $m_{v, i}$ is 1 .

The following lemma is similar to [Evertse 1984, Lemma 5]. Let $S_{\infty}$ and $T_{\infty}$ be respectively the set of all infinite places in $k$ and $L$, also, let $s_{\infty}=\# S_{\infty}$ and $t_{\infty}=\# T_{\infty}$. Note that every place in $T_{\infty}$ is complex, and hence

$$
t_{\infty}=[L: \mathbb{Q}] / 2 \leq 4 m d .
$$

For a real number $B$ with $0<B<1$, put

$$
R(B)=(1-B)^{-1} B^{B /(B-1)} .
$$

Lemma 4.7. Let $B$ be a real number with $1 / 2 \leq B<1$. There exists a set $\mathscr{W}_{1}$ of cardinality at most

$$
5^{s^{\prime}+s_{1}+s_{m}-s_{\infty}} \times 3^{t_{\infty}} \times R(B)^{s^{\prime}+s_{1}+2 s_{m}-s_{\infty}+t_{\infty}-1},
$$

consisting of tuples $\left(\left(\rho_{V}\right)_{V \in T},\left(\Gamma_{V}\right)_{V \in T}\right)$ with $\rho_{V}^{3}=1$ and $\Gamma_{V} \geq 0$ for $V \in T$ and $\sum_{V \in T} \Gamma_{V}=B$ with the following property: for every $\zeta \in \mathscr{V}^{1}$ there is a tuple $\left(\left(\rho_{V}\right)_{V \in T},\left(\Gamma_{V}\right)_{V \in T}\right) \in \mathscr{W}_{1}$ such that $\zeta$ satisfies

$$
\min \left(1,\left|1-\rho_{V} \zeta\right|_{V}\right) \leq\left(8 A h(\zeta)^{-3}\right)^{\Gamma_{V}}, \text { for } V \in T
$$


Proof. Consider the index set

$$
I=\left\{(w, j) \mid\left(j=1, w \in\left(S^{\prime} \cup S_{1} \cup T_{\infty}\right) \backslash\left(S_{m} \cup S_{\infty}\right)\right) \text { or }\left(j=1,2, w \in S_{m}\right)\right\} .
$$

Then $\# I \leq q:=s^{\prime}+s_{1}+2 s_{m}-s_{\infty}+t_{\infty}$. For $\zeta \in \mathscr{V}^{1}$ and $(w, j) \in I$, let

$$
m_{w, j}= \begin{cases}m_{v} & \text { if } w=v \in\left(S^{\prime} \cup S_{1}\right) \backslash\left(S_{m} \cup S_{\infty}\right), \\ m_{V} & \text { if } w=V \in T_{\infty}, \\ m_{v, 1} & \text { if } w=v \in S_{m} \text { and } j=1, \\ m_{v, 2} & \text { if } w=v \in S_{m} \text { and } j=2 .\end{cases}
$$

By Lemma 4.2, we have

$$
\prod_{(w, j) \in I} m_{w, j} \leq 8 A h(\zeta)^{-3}, \text { for } \zeta \in \mathscr{V}^{1}
$$

We know form [Evertse 1984, Lemma 4] that there exists a set $\mathcal{W}$ of cardinality at most $R(B)^{q-1}$ consisting of tuples $\left(\Phi_{w, j}\right)_{(w, j) \in I}$ such that for every $\zeta \in \mathscr{V}^{1}$ there is a tuple $\left(\Phi_{w, j}\right)_{(w, j) \in I}$ such that

$$
m_{w, j} \leq\left(8 A h(\zeta)^{-3}\right)^{\Phi_{w, j}}
$$

Here the tuples can be chosen such that if $m_{w, j}=1$, then $\Phi_{w, j}=0$. In particular, if $T_{v}^{j}$ is empty, we put $\Phi_{w, j} / t_{v}^{j}=0$. We define

$$
\Gamma_{V}= \begin{cases}0 & \text { if } V \in T_{v}^{0} \text { for some } v \in S^{\prime} \cup S_{1} \cup S_{m} \backslash S_{\infty}, \\ \Phi_{w, 1} / t_{v}^{1} & \text { if } V \in T_{v}^{1} \text { for some } v \in\left(S^{\prime} \cup S_{1} \cup S_{m}\right) \backslash S_{\infty}, \\ \Phi_{w, 2} / t_{v}^{2} & \text { if } V \in T_{v}^{2} \text { for some } v \in S_{m} \\ \Phi_{w, j} & \text { if } V \in T_{\infty} .\end{cases}
$$

Then inequality (4-4) holds. By Lemma 4.6, there are at most $5^{s^{\prime}+s_{1}+s_{m}-s_{\infty}} \times 3^{t_{\infty}}$ choices of $\rho_{V}$ 's.

Now take $B=0.846$. The total number of $\zeta \in W^{1}$ that satisfy a fixed system (4-4) and for which we have $h(\zeta) \geq e^{8} / 2$ is at most 25 (see [Evertse 1984, p. 583]). The cardinality of $\mathcal{W}^{1}$ is at most

$$
\begin{aligned}
5^{s^{\prime}+s_{1}+s_{m}-s_{\infty}} & \times 3^{t_{\infty}} \times R(B)^{s^{\prime}+s_{1}+2 s_{m}-s_{\infty}+t_{\infty}-1} \\
& \leq 5^{s^{\prime}+s_{1}+s_{m}-s_{\infty}} \times 3^{t_{\infty}} \times(49 / 3)^{s^{\prime}+s_{1}+2 s_{m}-s_{\infty}+t_{\infty}-1} \\
& \leq 2 / 25 \times(3 / 49) \times(245 / 3)^{s^{\prime}+s_{1}} \times(12005 / 9)^{s_{m}} \times(3 / 245)^{s_{\infty}} \times(7)^{2 t_{\infty}} .
\end{aligned}
$$

We note that $t_{\infty}$ is at most $4 m d$. A simple calculation shows that

$$
\# W^{1} \leq 2 / 25 \times(3 / 49) \times 7^{2.27\left(s^{\prime}+s_{1}\right)+3.7 s_{m}+8 m d} \times(3 / 245)^{s_{\infty}}
$$


By [Evertse 1984, (36)], we have $h(\lambda x / \mu y) \leq 2 h(\zeta(x, y))$. All of this yields the following lemma.

Lemma 4.8. The total number of $(P, Q) \sim\left(P_{l}, Q_{l}\right)$ with $Q=(f, g)$ such that $h((f-\alpha) /(f-\beta)) \geq e^{8}$ is at most

$$
6 / 49 \times 7^{2.27\left(s^{\prime}+s_{1}\right)+7.2 s_{m}+8 m d} \times(3 / 245)^{s_{\infty}} .
$$

Proof of Theorem 1.3. We first fix the equivalence class of $\left(P_{l}, Q_{l}\right)$. We follow the argument in [Evertse 1984, p. 583]. Let $\tilde{s}=\# \tilde{S}$. The group of $\tilde{S}$-units is the direct product of $\tilde{s}$ multiplicative cyclic groups, one of which is finite. The fraction $(f-\alpha) /(f-\beta)$ is a $\tilde{S}$-unit. We assume that for each $v \in S^{\prime} \cup S_{1} \cup S_{m} \backslash S_{\infty}$, a place $V_{v} \in T_{v}$ is chosen. Consider the index set

$$
\Phi:=\left\{\left(i_{v}\right)_{v} \mid i_{v}=1,2,3,4,5, v \in S^{\prime} \cup S_{1} \cup S_{m} \backslash S_{\infty}\right\}
$$

For each $\phi=\left(i_{v}\right)_{v} \in \Phi$, let

$$
\mathscr{V}_{\phi}^{1}=\left\{\zeta \in \mathscr{V}^{1} \mid \zeta \text { is of type } i_{v} \text { at every } v \in S^{\prime} \cup S_{1} \cup S_{m} \backslash S_{\infty}\right\}
$$

Then by (2-1) and (4-3), under the map

$$
\begin{aligned}
\mathscr{V}^{1} & \rightarrow \prod_{V \in \tilde{S} \backslash \tilde{S}_{\infty}} K_{V}^{*} \\
\zeta & \mapsto\left(|(f-\alpha) /(f-\beta)|_{V}\right)_{V},
\end{aligned}
$$

the image of each $\mathscr{V}_{\phi}^{1}$ is in a coset of a subgroup which is a direct product of less than $s^{\prime}+s_{1}+s_{m}-s_{\infty}$ multiplicative cyclic groups. This shows that, for a fixed $\phi$, the set of all $(f-\alpha) /(f-\beta)$ for which $\zeta \in \mathscr{V}_{\phi}^{1}$ is in a coset of a subgroup which is a direct product of less than $s_{3}:=t_{\infty}+s^{\prime}+s_{1}+s_{m}-s_{\infty}$ multiplicative cyclic groups. Let $n$ be a positive integer. Then there is an $\tilde{S}$-unit $z$ and an element $\omega \in K$ belonging to a fixed set of cardinality at most $n^{s_{3}}$ which does not depend on $f$ such that $(f-\alpha) /(f-\beta)=\omega z^{n}$. Let $\omega$ be a fixed element of this set and let $\theta$ be a fixed $n$ 'th root of $\omega$. By [Evertse 1984, Lemma 1], the number of nonzero $z$ in $K$ with $h(\theta z)<e^{8 / n}$ is at most $5\left(2 e^{24 / n}\right)^{[K: \mathbb{Q}]}$. Also, the fraction $(f-\alpha) /(f-\beta)$ determines $\zeta$. Using these and taking $n=49 / 3$, we see that the cardinality of the subset of $\mathscr{V}^{1}$ consisting of those $\zeta$ with $h((f-\alpha) /(f-\beta))<e^{8}$ is at most

$$
\begin{aligned}
5^{s^{\prime}+s_{1}+s_{m}-s_{\infty}} \times 5 n^{s_{3}}\left(2 e^{24 / n}\right)^{[K: \mathbb{Q}]} & \leq(245 / 3)^{s^{\prime}+s_{1}+s_{m}-s_{\infty}} \times 5 \times(49 / 3)^{t_{\infty}} \times 8.78^{4 m d} \\
& \leq 5 \times 7^{2.27\left(s^{\prime}+s_{1}+s_{m}\right)+10.3 m d} \times(3 / 245)^{s_{\infty}} .
\end{aligned}
$$


Therefore,

$$
\begin{aligned}
\# \mathscr{C} \leq & 4 \times|E(k) / 24 E(k)| \times(3 / 245)^{s_{\infty}} \times\left(6 / 49 \times 7^{2.27\left(s^{\prime}+s_{1}\right)+3.7 s_{m}+8 m d}\right. \\
& \left.+3 / 49 \times 7^{2.27\left(s^{\prime}+s_{1}+s_{m}\right)+10.3 m d}\right) \\
\leq & 4 \times\left|E(k)_{\mathrm{tor}} / 24 E(k)_{\mathrm{tor}}\right| \times(3 / 245)^{s_{\infty}} \times 24^{r} \times 6 \times 7^{2.27\left(s^{\prime}+s_{1}\right)+3.7 s_{m}+10.3 m d} \\
\leq & 4 \times 6 \times\left|E(k)_{\mathrm{tor}} / 24 E(k)_{\mathrm{tor}}\right| \times(3 / 245)^{s_{\infty}} \times 7^{1.64 r+2.27\left(s^{\prime}+s_{1}\right)+3.7 s_{m}+10.3 m d} .
\end{aligned}
$$

The map $\mathscr{b} \rightarrow E\left(\mathbb{O}_{S}\right)$ given by $(P, Q) \mapsto P$ is 4 to 1 . If $s_{\infty} \geq 2$, then

$$
6 \times\left|E(k)_{\text {tor }} / 24 E(k)_{\text {tor }}\right| \times(3 / 245)^{s_{\infty}} \leq 6 \times 24^{2} \times(3 / 245)^{2}<1,
$$

and the theorem is proved. Otherwise, the number field $k$ has degree at most 2 , and the order of the torsion part of the multiplicative group $k^{*}$ is at most 6 . In this case, via Weil pairing, we see that if $E(k)_{\text {tor }}$ contains a subgroup of the form $\mathbb{Z} / N \mathbb{Z} \times \mathbb{Z} / N \mathbb{Z}$ then $N \leq 6$. Consequently, we have $\left|E(k)_{\text {tor }} / 24 E(k)_{\text {tor }}\right| \leq 24 \times 6$ and hence

$$
6 \times\left|E(k)_{\mathrm{tor}} / 24 E(k)_{\mathrm{tor}}\right| \times(3 / 245)^{s_{\infty}} \leq 36 \times 24 \times(3 / 245)<11,
$$

as we wished to show.

\section{References}

[Chi et al. 2004] W.-C. Chi, K. F. Lai, and K.-S. Tan, "Integral points on elliptic curves over function fields”, J. Aust. Math. Soc. 77:2 (2004), 197-208. MR 2005g:11093 Zbl 02158910

[Chi et al. $\geq 2006$ ] W.-C. Chi, P.-Y. Huang, and K.-S. Tan, "Uniform bounds for the number of integer solutions to $Y^{n}=f(X)$ ". In preparation.

[Evertse 1984] J.-H. Evertse, "On equations in S-units and the Thue-Mahler equation", Invent. Math. 75:3 (1984), 561-584. MR 85f:11048 Zbl 0521.10015

[Gross and Silverman 1995] R. Gross and J. Silverman, "S-integer points on elliptic curves", Pacific J. Math. 167:2 (1995), 263-288. MR 96c:11057 Zbl 0824.11038

[Hindry and Silverman 1988] M. Hindry and J. H. Silverman, "The canonical height and integral points on elliptic curves”, Invent. Math. 93:2 (1988), 419-450. MR 89k:11044 Zbl 0657.14018

[Lang 1978] S. Lang, Elliptic curves: Diophantine analysis, Grundlehren der Mathematischen Wissenschaften 231, Springer, Berlin, 1978. MR 81b:10009 Zbl 0388.10001

[Silverman 1984] J. H. Silverman, "The $S$-unit equation over function fields", Math. Proc. Cambridge Philos. Soc. 95:1 (1984), 3-4. MR 85e:11018 Zbl 0533.10013

[Silverman 1986] J. H. Silverman, The arithmetic of elliptic curves, Graduate Texts in Mathematics 106, Springer, New York, 1986. MR 87g:11070 Zbl 0585.14026

[Silverman 1987] J. H. Silverman, "A quantitative version of Siegel's theorem: integral points on elliptic curves and Catalan curves”, J. Reine Angew. Math. 378 (1987), 60-100. MR 89g:11047 Zbl 0608.14021

[Tan 2002] K.-S. Tan, “A 2-division formula for elliptic curves”, National Taiwan University, January 2002. 
Received April 19, 2004.

\section{WEN-CHEN CHI}

DEPARTMENT OF MATHEMATICS

NATIONAL TAIWAN NORMAL UNIVERSITY

TAIPEI

TAIWAN

wchi@math.ntnu.edu.tw

KING FAI LAI

SCHOOL OF MATHEMATICS AND STATISTICS

UNIVERSITY OF SYDNEY

SYDNEY, NSW 2006

AUSTRALIA

kflai@maths.usyd.edu.au

KI-SENG TAN

DePARTMENT OF MATHEMATiCS

NATIONAL TAIWAN UNIVERSITY

TAIPEI

TAIWAN

tan@math.ntu.edu.tw 